\title{
ARDEP, a Rapid Degenerate Primer Design Pipeline Based on $k$-mers for Amplicon Microbiome Studies
}

\author{
Yueni Wu ${ }^{1,2}$, Kai Feng ${ }^{1,2}$, Ziyan Wei ${ }^{3}$, Zhujun Wang ${ }^{1,2}$ and Ye Deng ${ }^{1,2,3, *(1)}$ \\ 1 Key Laboratory for Environmental Biotechnology, Research Center for Eco-Environmental Sciences, \\ Chinese Academy of Sciences, Beijing 100085, China; ynwu_st@rcees.ac.cn (Y.W.); \\ kaifeng_st@rcees.ac.cn (K.F.); zjwang_st@rcees.ac.cn (Z.W.) \\ 2 College of Resources and Environment, University of Chinese Academy of Sciences, Beijing 100049, China \\ 3 Institute of Marine Science and Technology, Shandong University, Qingdao 266237, China; w.ziyan@163.com \\ * Correspondence: yedeng@rcees.ac.cn; Tel.: +86-10-62840082
}

Received: 17 July 2020; Accepted: 14 August 2020; Published: 17 August 2020

check for updates

\begin{abstract}
The survey of microbial diversity in various environments has relied upon the widespread use of well-evaluated amplification primers for taxonomic marker genes (e.g., prokaryotic $16 \mathrm{~S}$ and fungal ITS). However, it is urgent to develop a fast and accurate bioinformatic program to design primers for microbial functional genes to explore more mechanisms in the microbial community. Here, we provide a rapid degenerate primer design pipeline (ARDEP) based on the $k$-mer algorithm, which can bypass the time-consuming step of sequence alignment to greatly reduce run times while ensuring accuracy. In addition, we developed an open-access platform for the implementation of primer design projects that could also calculate the amplification product length, GC content, Annealing Temperature (Tm), and $\Delta G$ of primer self-folding, and identify covered species and functional groups. Using this new platform, we designed primers for several functional genes in the nitrogen cycle, including nap $A$ and amoA. Our newly designed primers achieved higher coverage than the commonly used primers for all tested genes. The program and the associated platform that applied the $k$-mer algorithm could greatly enhance the design and evaluation of primers for environmental microbiome studies.
\end{abstract}

Keywords: bioinformatic program; primer design; $k$-mer; analysis platform; primer assessment

\section{Introduction}

At present, studies of environmental microbial community composition and diversity generally follow the procedure of sample collection, DNA extraction, Polymerase Chain Reaction (PCR) amplification, and high-throughput sequencing [1]. PCR amplification and high-throughput sequencing have greatly changed our understanding of microbial diversity in various environments by bypassing the need for cultivation [2]. Amplicon sequencing is a highly targeted method for analyzing gene or species variations across broad taxonomic ranges [3], using primers to amplify specific regions within environmental DNA to enrich the targeted sequences [4]. Thereafter, high-throughput sequencing is carried out to capture the genetic diversity of the amplified products. Since PCR is the central step of this procedure, the design of primers to amplify the target gene is the key step to determine the specificity and coverage of amplicon sequencing for environmental microbiome studies. The amplification primers of marker genes, such as the commonly used primers for prokaryotic 16S rRNA gene and fungal Internal Transcribed Spacer (ITS) region, which are often used in diversity surveys, have been widely used and evaluated [5,6]. However, with recent expansions in sequencing capabilities due to next-generation sequencing, the existing primers for other functional genes are unable to cover 
broad taxonomic groups with their variable sequences. Only by designing suitable primers can PCR reactions for the detection of taxonomic and functional genes be carried out accurately.

Coverage and specificity are both extremely important issues for the quality of primers. The coverage of a primer, that is, the proportion of sequences within a given sequence set that is matched, can be improved by introducing nucleic degeneracy, however, higher nucleic degeneracies in a primer might negatively affect its specificity. Thus, balancing the nucleic degeneracy is also important for the primer design in microbiome studies. In addition, current primer design methods are mainly based on aligned DNA sequences. The most highly conserved region of a target gene generally has a similar sequence across many different species. Thus, high-coverage primers could be designed in these conserved regions [7]. Many software programs for the design of degenerate primers are based on this pattern, including HYDEN [7], PrimerProspector [8], and DegePrime [9]. However, with the rapidly increasing number of sequences deposited in public databases, the time cost of multiple sequence alignment has vastly increased. With high requirements for sequence length and quality, the accurate alignment of thousands of sequences may take several days, while tens of thousands of sequences may run for dozens of days [10]. Therefore, there is an acute need for a new, fast, and accurate method to design primers for amplicon sequencing technology, especially for targeting microbial functional genes.

The $k$-mer algorithm is a commonly used algorithm to improve assembly effect in the analysis of metagenomic sequencing results [11]. It divides the sequence into a string containing $k$ bases. Generally, a sequence with the length of $\mathrm{m}$ can be divided into $m-k+1 k$-mers. Using the sequence AACTGACTGA as an example: When $k=4$, for the input sequence, we start from the first base and use the form of sliding window (step size is 1) to extract the $4 \mathrm{bp}$ sequence successively. This sequence can be divided into 7 -mers: AACT, ACTG, CTGA, TGAC, GACT, ACTG, and CTGA. At present, although the $k$-mer algorithm is widely used in bioinformatics, such as genome assembly, genome sequencing coverage evaluation, error correction of sequencing data, multiple sequence alignment, and repeat sequence detection, it has not as yet been used in the development of primer design methods, especially for the design of degenerate primers. Because the conserved region of a gene should be the same or very similar across multiple sequences, we infer that the frequent $k$-mers should originate from those regions and be detectable when the $k$ value is greater than a certain length. In view of this, we provided a new primer design method based on the $k$-mer algorithm, which can avoid the tedious step of sequence alignment and greatly improve the running speed while still ensuring accuracy. This paper provided a theoretical guidance for a new idea of microbial functional gene amplification primer design. Based on this algorithm, we designed degenerate primers for several microbial functional genes, and verified their coverage and specificity.

\section{Materials and Methods}

\subsection{Analysis Platform of ARDEP}

We developed a bioinformatic platform for our rapid degenerate primer design program (ARDEP, available at http://mem.rcees.ac.cn:8082/) for amplicon microbiome studies, to which users may upload sequence databases for primer design and assessment. The analysis platform allows for sequence length statistics, length filtering, and redundancy removal to be performed on the sequence database using tools located in the "Sequence Processing" section. Within this section, "Length statistics" provides quick information about sequence length distribution. "Trim by Sequence Length" is a program to trim sequences based on length. Only sequences longer than (or equal to) the minimum length and shorter than (or equal to) the maximum length are retained. Users are able to choose between either deleting the longer sequences or trimming them to the required length. "Remove same sequences for FASTA" is for the removal of same exact sequences from the database. After performing sequence database quality control, users may run ARDEP for primer design in the "Primer Processing" section. The tools within this section also allow users to evaluate primers. "Coverage Calculator" could calculate 
primer coverage using Nucleotide Basic Local Alignment Search Tool (BLASTn). "Amplification Product Length" allows for calculation of amplification product length. If the uploaded database contains taxonomic information, "Covered Taxonomy Calculator" can be used to count the sequence number of taxonomy and functional group covered by primer sets. This tool not only counts the covered sequence number of taxonomy, but also assesses covered types of gram-positive/negative and oxygen requirement as based on the BugBase database [12].

\subsection{Algorithm of ARDEP}

\subsubsection{Division of Database Sequences by $k$-mer Program}

Based on the $k$-mer algorithm, primer length is set as $k$, and all sequences are divided into $k$-mers (Figure 1). If $k$ is set as a length range $\left(\mathrm{k}_{1}<k<\mathrm{k}_{1+\mathrm{n}}\right)$, then $n$ times will be calculated according to different $k$ values. The $k$-mer statistics are calculated using jellyfish software [13].

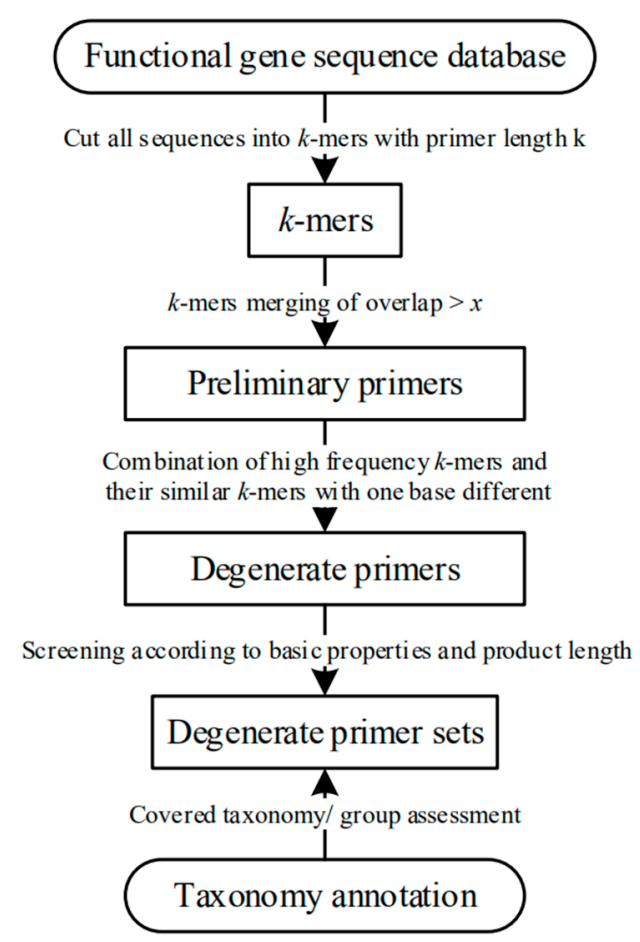

Figure 1. Workflow for primer design process.

\subsubsection{Degenerate Primer Combination}

All $k$-mers with overlap $>x$ will be combined because $k$-mers with overlap $>x$ are defaulted to exist in the position of primer length- $x$ base difference. The value $x$ is set by the user, with the default value set as half of the primer length. The $k$-mer with the highest frequency is retained. If the frequency of two $k$-mers' overlap $>x$ are the same, the longer $k$-mer will be kept. For example, the frequency of one $k$-mer CGCGGTTGCTCGTGTGG is 1218, and the frequency of the other k-mer GTTGCTCGTGGCGCG is 1137 . By default, these two $k$-mers are located four bases apart. Therefore, only CGCGGTTGCTCGTGTGTGG will be retained. According to how frequently a $k$-mer appears, potentially dozens of high-frequency $k$-mers could be selected for downstream processing. The $k$-mers which contain 1 base different from the high-frequency $k$-mers are combined with the high-frequency $k$-mer in the form of a degenerate base. The preliminary primers have been designed. For example, searching for $k$-mers of coverage $>1 \%$ and with a base difference between the high frequency $k$-mer ACATGCCAATGCTGGT, we find two $k$-mers, ACATGCGATGCTGGT and ATATGCCAATGCTGGT. These can be combined in the form of degenerate bases to AYATGCCRATGCTGTGT (Y: C or T, R: 
A or $\mathrm{G})$. According to the amplification product fragment length and coverage, the most suitable primer sets for the database are selected.

\subsubsection{Calculation of Basic Primer Properties}

- The annealing temperature $(\mathrm{Tm})$, secondary structure ( $\Delta \mathrm{G}$ of primer's self-folding), and GC content $(\mathrm{GC} \%)$ of primers are calculated as follows:

- $\mathrm{GC} \%=(\mathrm{G}+\mathrm{C}) /$ primer length

- Annealing temperature (Tm): when the primer length is shorter than $20 \mathrm{bp}: \mathrm{Tm}=4(\mathrm{G}+\mathrm{C})$ $+2(\mathrm{~A}+\mathrm{T})$; when the primer length is longer than $20 \mathrm{bp}, \mathrm{Tm}=62.3^{\circ} \mathrm{C}+0.41{ }^{\circ} \mathrm{C}(\mathrm{GC} \%)-$ 500/primer length.

- The stability parameter $\Delta \mathrm{G}$, which is the free energy needed for DNA double-strand formation, reflects the stability of the double-strand structure. If the complementary base of the primer is too long, a primer dimer or hairpin structure could be produced, which leads to high $\Delta G$ and the PCR reaction cannot proceed normally. This parameter is calculated by mfold software [14].

\subsubsection{Coverage Verification and Covered Taxonomy/Group Calculation}

The primer coverage and amplification product fragment length are calculated, and the main groups that the primer set could cover are detected, including covered taxonomy, oxygen requirement type, and gram type (i.e., gram-positive and gram-negative). The classifications of oxygen requirement and gram type are based on the species phenotype database of BugBase [12].

\subsubsection{Parameters Required by ARDEP Platform}

- Number of database sequences;

- Primer length (maximum and minimum, default 18-20 bp);

- Amplification product fragment length (maximum and minimum, default value is 200-500 bp);

- Minimum coverage users could accept of the output primer (the default value is $20 \%$, i.e., 0.2 );

- Overlap of high-frequency $k$-mers to be merged (the default value is 10 );

- $\quad$ DNA folding temperature in PCR experiment (the default value is $57^{\circ} \mathrm{C}$ );

- Ionic conditions of $\mathrm{Mg}++$ (the default value is 20) and $\mathrm{Na}+$ (the default value is 0 ) in PCR experiment (Figure 2).

\begin{tabular}{|l|}
\hline ARDEP A rapid degenerate primer design program. (Galaxy Version 1.0.0) \\
Database file(FASTA) \\
\hline Total number of database sequences \\
\hline The Minimum Primer Length \\
\hline 17 \\
\hline The Maximum Primer Length \\
\hline 19 \\
\hline The Minimum Amplification Product Length \\
\hline 200 \\
\hline The Maximum Amplification Product Length \\
\hline 500 \\
\hline Remove Unique Kmer \\
\hline 0.01 \\
\hline Minimum Coverage \\
\hline 0.02 \\
\hline Minimum Bases for Similar Kmer Merge \\
\hline 10 \\
\hline Folding Temperature (between $\mathbf{0}$ and 100) \\
\hline 57 \\
\hline Ionic conditions: [Na+ $]$ \\
\hline 0 \\
\hline Ionic conditions: [Mg++ \\
\hline 20 \\
\hline \\
\hline \\
\hline
\end{tabular}

Figure 2. The $k$-mer-based primer design tool interface. The default values for program operation parameters are shown in the figure. 


\subsection{Test Datasets}

We chose the denitrification gene napA during the nitrogen cycle to test the ARDEP primer design tool. The napA gene could encode proteins that catalyze the reduction of $\mathrm{NO}_{3}{ }^{-}$to $\mathrm{NO}_{2}{ }^{-}$ (periplasmic-bound nitrate reductase). In order to design reliable primers for the nap $A$ gene, the marker gene of dissimilatory nitrate reductase in the dissimilatory nitrogen reduction to ammonia (DNRA) process, we collected a credible napA functional gene database. Based on 472 napA sequences that were retrieved from GeoChip probe database [15], we screened for sequences with lengths of 500-1000 bp. These sequences were aligned using the Muscle program [10] and their similarity was calculated. Only the sequences with similarity $>80 \%$ were retained and duplicate sequences were cut such that only one sequence per species was kept. Finally, 126 highly conserved sequences were chosen as seed sequences. Using HMMER, we searched for the conserved domain of homologous proteins in the GenBank Protein Database [16,17], gathering all homologous sequences which could be annotated as napA. Redundant sequences and unqualified sequences were removed, resulting in 38,768 sequences being retained. According to the $E$-value in HMMER $\left(E<10^{-5}\right)$ and the annotation information of sequences (must include at least one set of "periplasic nitrate reduce, nap $A$ gene product, nap $A$, nitrate reduce, nitrate oxidoreductase" as key words), we selected 3397 highly credible sequences from the collected homologous sequences. On this basis, we downloaded the corresponding protein and nucleic sequences according to the protein accession number. Through the taxid corresponding to the protein accession number, we supplemented the taxonomy information to complete the database construction. These sequences and their annotation were used to test the ARDEP primers design tool. In order to calculate the specificity of the designed primers for the napA gene, we constructed the narG gene database which also reduced $\mathrm{NO}_{3}{ }^{-}$to $\mathrm{NO}_{2}{ }^{-}$(membrane-bound nitrate reductase). The construction of the nar $G$ sequence database was based on 47 seed sequences. Similar to nap $A$ we searched for the conserved domain of homologous proteins in the GenBank Protein Database through HMMER [16], gathering all homologous sequences which may be annotated as nar $G\left(E<10^{-5}\right.$ while including at least one set of "nitrate reductase, nitrate-reductase, dissimilatory membrane-bound nitrate reductase, nar $G$ gene product, nitrate reductase alpha subunit, respiratory nitrate reductase, $n a r G$, molybdopterin" as key words). Thus, 30,217 sequences were retained after removal of redundant and unqualified sequences. On the other hand, the specificity was also evaluated based on the NCBI nt database $(58,992,497$ sequences, updated date: 6 June 2020) to rule out the possibility of primers overwriting other homologous or similar genes.

To test the applicability of our method to a relatively large database, we downloaded 119,096 sequences from NCBI and 323,085 sequences from FunGene [18] that were annotated as $a m o A$. FunGene is a free database containing redundant sequences of a number of functional genes of microorganisms. These $a m o A$ sequences were merged to remove redundant sequences, and 173,790 sequences with length of 200-1000 bp were retained. On the other hand, we built ammonia-oxidizing archaea (AOA) and ammonia-oxidizing bacteria (AOB) databases based on 52 seed sequences through HMMER [16]. All homologous sequences were gathered which may be annotated as amo $A$ ( $E<10^{-5}$ while including at least one set of "ammonia monooxygenase, ammonia monooxygenase subunit $\mathrm{A}$, $a m o A$ gene product, putative ammonia monooxygenase subunit $\mathrm{A}$, amo $A$ " as key words). After removing redundancy, these sequences of 350-650 bp length were divided into AOA and AOB according to their taxonomy annotation; 45,771 AOA sequences and 42,659 AOB sequences were used to design $\mathrm{AOA}$ and $\mathrm{AOB}$ primers by ARDEP, respectively. In order to calculate the specificity of the designed primers for the amo $A$ gene, a total of 22,357 nonredundant $p m o A$ sequences were collected from NCBI, including 1165 archaea sequences and 21,192 bacteria sequences. The $p m o A$ gene is a homologous gene of $a m o A$, which is, however, involved in completely different ecological processes. On the other hand, the specificity was also evaluated based on the NCBI nt database to rule out the possibility of primers overwriting other homologous or similar genes. 


\section{Results}

\subsection{Primer Design of Functional Gene napA}

The nap A sequence database included 3397 nonredundant nap $A$ gene sequences of high credibility and identified species. These sequences were mainly composed of Proteobacteria, accounting for $71.65 \%$ of the sequences, while the remaining were $14.72 \%$ Firmicutes, $12.42 \%$ Actinobacteria, and $1.21 \%$ unclassified phyla. Based on BugBase classification [12], 52.46\% of the sequences were classified as facultative anaerobic, $33.41 \%$ were classified as aerobic, and $2.24 \%$ were classified as anaerobic. According to the gram classification, $71.77 \%$ of the sequences were classified as gram-negative, while $26.58 \%$ were classified as gram-positive bacteria. At present, the most widely used primers for the nap A gene are v16cf-GCNCCNTGYMGNTTYTGYGG/v17cr-RTGYTGRTTRANANCCCATTCCA, which could only cover less than $1 \%$ of the sequences in this database, with an amplicon length of 1040 bp. In order to design primers with higher coverage and an assignable amplification product fragment length for high-throughput sequencing, we used ARDEP to design new primers based on our nap $A$ sequence database. Through the $k$-mer algorithm, all sequences in the database were cut into $k$-mers with the lengths from 19 to $20 \mathrm{bp}$. The basic properties of primers were calculated after combining degenerate bases. The ARDEP output file of individual primers and their basic properties is shown in Table S1. The primer named as napA_kmer5 (TTYTAYGACTGGTAYKSYGA) possessed the highest coverage, covering $80.01 \%$ of all sequences. The top three primers with the highest coverage and their basic properties are listed in Table 1.

Table 1. napA primers with basic properties.

\begin{tabular}{|c|c|c|c|c|c|c|c|c|c|}
\hline \multirow[b]{2}{*}{ Primer Name } & \multirow[b]{2}{*}{ Primer Sequence } & \multirow{2}{*}{$\begin{array}{l}\text { Product } \\
\text { Length }\end{array}$} & \multirow{2}{*}{\multicolumn{2}{|c|}{ Coverage }} & \multicolumn{2}{|c|}{ Specificity } & \multicolumn{3}{|c|}{ Primer Properties } \\
\hline & & & & & narG & nt & $\Delta \mathrm{G}$ & GC\% & $\mathrm{Tm}$ \\
\hline napA_kmer5 & TTYTAYGACTGGTAYKSYGA & \multirow{2}{*}{421} & $80.01 \%$ & \multirow{2}{*}{$65.50 \%$} & \multirow{2}{*}{$0.82 \%$} & \multirow{2}{*}{$<0.01 \%$} & $(-2.07)-(1.70)$ & $30.00-55.00 \%$ & $52.00-62.00$ \\
\hline napA_kmer8 & ACSTGGGADAYNCADCADAC & & $67.91 \%$ & & & & $(-3.15)-(2.10)$ & $40.00-65.00 \%$ & $56.00-66.00$ \\
\hline napA_kmer6 & TGGGGYGAVCADACNGAYGT & \multirow{2}{*}{466} & $77.77 \%$ & \multirow{2}{*}{$64.20 \%$} & \multirow{2}{*}{$0.80 \%$} & \multirow{2}{*}{$<0.01 \%$} & $(-1.65)-(1.24)$ & $45.00-70.00 \%$ & $58.00-68.00$ \\
\hline napA_kmer8 & ACSTGGGADAYNCADCADAC & & $67.91 \%$ & & & & $(-3.15)-(2.10)$ & $40.00-65.00 \%$ & $56.00-66.00$ \\
\hline
\end{tabular}

Based on the results of the preliminary design, primer sets were recommended according to the amplification product fragment length. An ARDEP output file of paired primers is shown in Table S2. According to the napA gene length and sequencing feasibility, we determined the optimal product length as 200-500 bp. Compared with the most used primer set v16cf/v17cr, the coverage of the napA gene was highly increased and the amplicon length was more optimal for high-throughput sequencing. Primer set napA_kmer5/napA_kmer8 with the highest coverage of $65.50 \%$ could amplify product with length $421 \mathrm{bp}$. Another primer set, napA_kmer6/napA_kmer8, with the coverage of $64.20 \%$ could amplify product with length $466 \mathrm{bp}$ (Table 1). Tm of forward and reverse primers of both primer sets was similar, which meant they were suitable to use in pairs. We carried out further assessment of these two primer sets and calculated their covered species, oxygen requirement, and gram type (Figure 3). Primer set napA_kmer5/napA_kmer8 could cover $87.59 \%$ Proteobacteria sequences, $18.00 \%$ Actinobacteria sequences, and $0.71 \%$ Firmicutes sequences (Figure 3a). Although the coverage of napA_kmer6/napA_kmer8 was a little lower than napA_kmer5/napA_kmer8, this primer set could cover more Proteobacteria sequences (89.44\%). However, it could barely cover Actinobacteria and Firmicutes sequences (Figure 3a). For oxygen demand types, napA_kmer5/napA_kmer8 could cover more aerobic sequences, while napA_kmer6/napA_kmer8 could cover more anaerobic sequences (Figure $3 b$ ). For gram types, both primer sets could cover more than $85 \%$ gram-negative sequences. However, napA_kmer5/napA_kmer8 could cover 10.30\% gram-positive sequences, while napA_kmer6/napA_kmer8 could barely cover any (Figure 3c). These two primer sets showed different coverage bias to our database. 


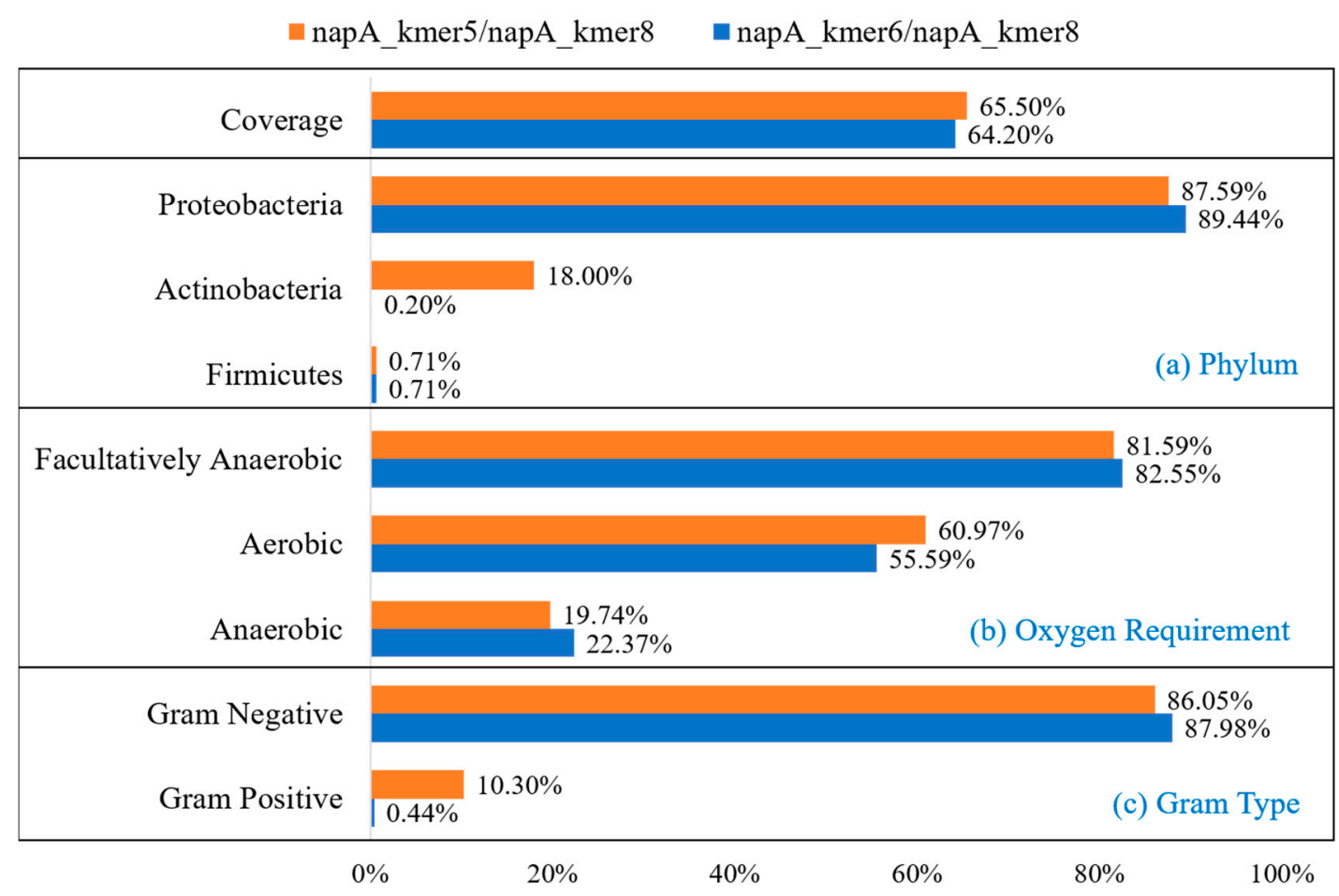

Figure 3. Covered sequence composition of two napA primer sets. (a) Phylum; (b) oxygen requirement type; (c) gram type.

The specificity of these primers was evaluated based on the NCBI nt database and narG database that we created (Table 1). The coverage of all these napA primers for the nt database was less than $0.01 \%$. In other words, these primers have quite good specificity to the napA gene. For the nar G database, the coverage of all designed primers was less than $0.82 \%$ (Table S2). In other words, our primers have good specificity even for homologous genes with similar sequences. The entire primer design process took a total of $13 \mathrm{~min}$. In the same computing environment, the computing time required for DegePrime, for primers designed with the highest coverage of $63.97 \%$ (TTYTAYGACTGGTAYTGCGA), was $1 \mathrm{~h}$ and $41 \mathrm{~min}$. The calculation time included MAFFT [19] sequence alignment calculation with maximum parallelism. Therefore, our method not only designed faster, but produced primers with higher coverage as well.

\subsection{Primer Design of Functional Gene amoA}

The most commonly used forward primer for AOA, Arch-amoA-for (CTGAYTGGGYTGGACATC), could cover $44.18 \%$ of AOA sequences in our database, while the reverse primer Arch-amoA-rev (TTCTTCTTTGTTGCCCAGATA) could cover $46.76 \%$ of AOA sequences. As a set, these primers could cover $41.17 \%$ of AOA sequences. The common forward primer for AOB, amoA_1F (GGGGHTTYTACTGGTGGT), could cover $50.07 \%$ of AOB sequences, while the reverse primer amoA_2R (CCCCTCKGSAAAGCCTTCTTC) could cover $54.77 \%$ of AOB sequences. Together as a set, the primers could only cover $43.97 \%$ of $\mathrm{AOB}$ sequences. We designed new primers for both $\mathrm{AOA}$ and $\mathrm{AOB}$ amoA sequence databases using ARDEP to see if we could get primers with higher coverage.

For 45,771 AOA sequences, we designed nine degenerate primer sets with coverage of over $50 \%$ (Table 2). The primer length was 19-20 bp and the amplification product fragment length was 200-500 bp. The coverage and basic properties of the individual primers were also output in the results (Table S3). AOA_kmer14 was the primer with the highest coverage at $88.99 \%$ of sequences, while AOA_kmer9 also reached a high coverage of $86.29 \%$. In addition, AOA_kmer4, AOA_kmer2, and AOA_kmer5 could cover more than $70 \%$ AOA sequences. The output file of AOA primer sets is shown in Table S4. The primer set AOA_kmer4/AOA_kmer14 showed the highest coverage at $66.07 \%$. 
This primer set could amplify products of $427 \mathrm{bp}$ length. We evaluated the specificity of these designed AOA primers using the pomA database we constructed. The coverage of all designed AOA primers to the $p m o A$ database was $<0.01 \%$. In other words, our AOA primers can hardly cover its homologous gene $p m o A$. The specificity of these AOA primers was also evaluated based on the NCBI nt database to see if they could cover other functional genes. The coverage of these primers for the nt database was less than $0.06 \%$ (Table 2). Compared with AOA database sequence number and primer coverage, we concluded that the specificity of these primers was reasonable for the archaea amo $A$ gene. The entire design process of ARDEP took $8 \mathrm{~h}$ and $3 \mathrm{~min}$.

Table 2. Recommended primer sets for amoA gene of AOA.

\begin{tabular}{ccccccc}
\hline \multicolumn{2}{c}{ Primer } & \multirow{2}{*}{ Coverage } & Product Length & \multicolumn{2}{c}{ Specificity } \\
\cline { 5 - 6 } Forward & Reverse & & Mode & pmoA & nt \\
\hline AOA_kmer4 & AOA_kmer14 & $66.07 \%$ & & 427 & $<0.01 \%$ & $<0.01 \%$ \\
AOA_kmer2 & AOA_kmer9 & $62.90 \%$ & 202 & $<0.01 \%$ & $0.06 \%$ \\
AOA_kmer2 & AOA_kmer5 & $56.08 \%$ & 433 & $<0.01 \%$ & $0.06 \%$ \\
AOA_kmer4 & AOA_kmer5 & $55.65 \%$ & 385 & $<0.01 \%$ & $0.06 \%$ \\
AOA_kmer8 & AOA_kmer14 & $55.18 \%$ & 472 & $<0.01 \%$ & $<0.01 \%$ \\
AOA_kmer2 & AOA_kmer15 & $52.71 \%$ & 407 & $<0.01 \%$ & $<0.01 \%$ \\
AOA_kmer4 & AOA_kmer6 & $52.14 \%$ & 211 & $<0.01 \%$ & $0.05 \%$ \\
AOA_kmer2 & AOA_kmer11 & $52.09 \%$ & 229 & $<0.01 \%$ & $<0.01 \%$ \\
AOA_kmer4 & AOA_kmer15 & $51.35 \%$ & 359 & $<0.01 \%$ & $<0.01 \%$ \\
\hline
\end{tabular}

For the 42,659 AOB sequences, we designed nine degenerate primer sets with coverage over $50 \%$ (Table 3). The primer length was 19-20 bp and the amplification product fragment length was 200-500 bp. AOB_kmer6 was the primer with the highest coverage at $84.12 \%$ of AOB sequences (Table S5). The output file of AOA primer sets is shown in Table S6. The primer set AOB_kmer3/AOB_kmer6 had the highest coverage at $55.50 \%$. We evaluated the specificity of these designed AOB primers using the pom $A$ database we constructed. The coverage of all designed AOB primers to the $p m o A$ database was less than $0.06 \%$. The specificity of these AOB primers was also evaluated based on the NCBI nt database. The coverage of these primers for the nt database was less than $0.05 \%$. Compared with AOB database sequence number and primer coverage, we concluded that the specificity of the designed primers was reasonable for the bacterial amo $A$ gene. The entire design process took $7 \mathrm{~h}$ and $47 \mathrm{~min}$.

Table 3. Recommended primer sets for amoA gene of AOB.

\begin{tabular}{|c|c|c|c|c|c|}
\hline \multicolumn{2}{|c|}{ Primer } & \multirow{2}{*}{ Coverage } & \multirow{2}{*}{$\begin{array}{c}\text { Product Length } \\
\text { Mode }\end{array}$} & \multicolumn{2}{|c|}{ Specificity } \\
\hline Forward & Reverse & & & $p m o A$ & nt \\
\hline AOB_kmer3 & AOB_kmer6 & $55.50 \%$ & 204 & $0.06 \%$ & $0.05 \%$ \\
\hline AOB_kmer1 & AOB_kmer22 & $53.66 \%$ & 439 & $<0.01 \%$ & $<0.01 \%$ \\
\hline AOB_kmer6 & AOB_kmer26 & $51.83 \%$ & 218 & $<0.01 \%$ & $<0.01 \%$ \\
\hline AOB_kmer3 & AOB_kmer17 & $51.63 \%$ & 204 & $0.06 \%$ & $<0.01 \%$ \\
\hline AOB_kmer1 & AOB_kmer5 & $51.30 \%$ & 448 & $<0.01 \%$ & $0.04 \%$ \\
\hline AOB_kmer6 & AOB_kmer22 & $51.12 \%$ & 294 & $<0.01 \%$ & $<0.01 \%$ \\
\hline AOB_kmer4 & AOB_kmer6 & $50.87 \%$ & 228 & $<0.01 \%$ & $0.04 \%$ \\
\hline AOB_kmer1 & AOB_kmer27 & $50.79 \%$ & 451 & $<0.01 \%$ & $<0.01 \%$ \\
\hline AOB_kmer17 & AOB_kmer26 & $50.10 \%$ & 218 & $<0.01 \%$ & $<0.01 \%$ \\
\hline
\end{tabular}

For all 173,790 nonredundant amoA sequences, including both AOA and AOB, we designed three degenerate primer sets with coverage of over 20\% (Table 4). The primer length was 18-20 bp, and amplification product fragment length was 200-500 bp. Among these primers, amoA_kmer15 and amoA_kmer20 could, individually, reach a coverage of more than $36 \%$. As AOA and AOB are often studied separately, the primer coverage designed based on the mixed database of the amo $A$ gene is 
relatively low. The specificity of these amoA primers was evaluated based on the NCBI nt database. The coverage of these primers for the nt database was less than $0.1 \%$ (Table 4 ). amoA_kmer7 could cover the most sequences in the nt database with the coverage of $0.06 \%$, namely 35,396 sequences. Compared with $a m o A$ database sequence number and primer coverage, we concluded that the specificity of the designed primers was reasonable for the $a m o A$ gene. For the homologous gene $p m o A$ which we pay special attention to, some primers showed certain preference. For example, the ability of amoA_kmer20 to cover the amoA gene is basically the same as pmoA. For the primer with poor specificity, it could be used with another primer with better specificity as well. For example, when paired with amoA_kmer6, the specificity of this primer set had become better. In this way, the amplification efficiency can also be guaranteed. The entire design process took $12 \mathrm{~h}$ and $55 \mathrm{~min}$.

Table 4. Recommended primers with basic properties for amoA.

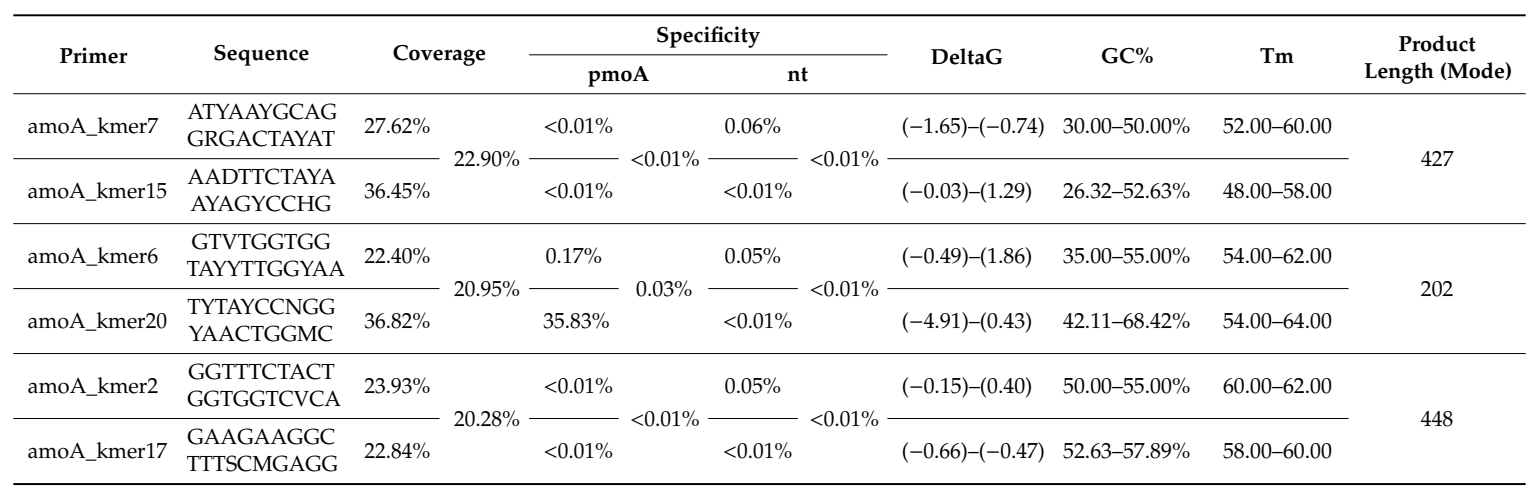

\section{Discussion}

With the development of molecular biology technology and metagenomic technology, amplicon sequencing has greatly changed our understanding of microbial diversity. PCR is one of the most basic and important tools in these technologies, and thus primer design and assessment have become key steps to ensure the accuracy of environmental detections [20,21]. The conventional programs for primer design across broad taxonomic ranges have all been based on aligned nucleic sequence [8,9]. However, the process of sequence alignment is highly time-consuming, with the alignment of thousands of sequences often taking dozens of hours. We have tested several sequence alignment programs in routine experiments and found that: (i) when more than 2000 nap $A$ sequences were aligned, the calculation time of MAFFT was $33 \mathrm{~h}$ and 23 min with less than 10 parallelism, and 45 min with maximum parallelism; (ii) when more than 4000 napA sequences were aligned, the calculation time of Clustal Omega [22] was $1 \mathrm{~h}$ and $33 \mathrm{~min}$, while MAFFT, with high accuracy, took more than $1 \mathrm{~h}$ with maximum parallelism; (iii) when more than 10,000 nirA gene sequences were aligned, the high-accuracy program MUSCLE took $88 \mathrm{~h}$ and $7 \mathrm{~min}$ (i.e., more than three days) while the fastest method Clustal Omega required $3 \mathrm{~h}$ and $5 \mathrm{~min}$ for the necessary calculations; (iv) when more than 30,000 amo $\mathrm{A}$ gene sequences were compared, MUSCLE required $156 \mathrm{~h}$ and $22 \mathrm{~min}$ (i.e., more than six days) while Clustal Omega took $4 \mathrm{~h}$ and $20 \mathrm{~min}$ for calculations (Table S7). These slow speeds greatly increase the time cost of primer design, and inaccurate alignments would likely decrease the possibility of successful primer searches in the conserved regions.

In order to shorten the calculation time of primer design, bypassing the sequence alignment seemed to be a logical route. The $k$-mer algorithm is often used to improve the assembly effect in the analysis of metagenomic sequencing results, but has never been used in primer design before. Theoretically, the conserved regions can be located for use as primers according to the frequency of $k$-mers without the need for alignment. Based on this, we designed a method for primer design with greatly improved calculation speed. Our results showed that the database calculation time of more than 3000 sequences was only about $13 \mathrm{~min}$ and for a database of more than 40,000 sequences, it took about $8 \mathrm{~h}$ each for $\mathrm{AOA}$ and $\mathrm{AOB}$ primer design. The calculation time for a database of over 
170,000 sequences was $12 \mathrm{~h}$ and $55 \mathrm{~min}$. Referring to the results of test data, we have created some rough statistics on the calculation time and data size. The calculation time included $k$-mer sequence division, degenerate primer merging, amplification product fragment length calculation, primer basic property calculation, covered group calculation, reading and writing files, and statistical calculations. There can be differences in the calculation time for the same sized dataset as calculation time is related to the degeneracy and sequence similarity.

The design and assessment of primers are closely related to how reliable the sequence database is. Therefore, choosing a credible and complete database is very important for primer design. For the napA gene database in our study, we used the method of protein conserved domain alignment to construct a database based on 472 nap $A$ sequences that were retrieved from the GeoChip probe database. According to the E-value in HMMER and the annotation information of sequences, we selected 3397 highly credible sequences from the collected homologous sequences from NCBI. These sequences are with high reliability and complete description information. For the functional genes of environmental microorganisms, numbers of sequence databases have already been completely built. In addition to the synthetic databases FunGene and Genbank mentioned in this article, there are many targeted databases, such as antibiotic resistance gene database SDARG [21], nitrogen cycling gene database NCycDB [23], et al. These targeted databases mostly carried out taxonomy annotation and reliability classification of sequences, which are also suitable for primer design and assessment. Our analysis platform could perform secondary screening on the length, redundancy, and other aspects of these database sequences. With high reliability of sequence database, the designed primers could exhibit good performance in real experiments. For $A O B$, we have performed an experiment to detect the diversity of soil AOB by amoA amplicons using designed AOB primer set AOB_kmer2/AOB_kmer28 (Table S6). From our preliminary amplification results (Figure S1), this designed primer set had high specificity in the tested soil samples, showing only one clear band in the proposed amplicon length ( 250 bp).

The advantage of ARDEP is not only reflected in the calculation speed. Compared with other primer design software, it provides more information. ARDEP allows users to provide a length range to design primers, rather than limiting them to a fixed length. ARDEP could also calculate the $\mathrm{GC} \%, \mathrm{Tm}$, and $\Delta \mathrm{G}$ of primers as a reference for experiments. It also provides recommendations for combinations of primer sets according to coverage and amplification product length. Meanwhile, most primer design software based on sequence alignments only provide the location of a single primer [9]. In addition, ARDEP could calculate the organisms covered by primer sets according to taxonomic annotations. This tool allows the user to be more specific in primer selection according to the studied environment. Our method provided theoretical guidance for the design and selection of microbial functional gene primers. However, the specific expression of primers in amplification still needs to be explained by PCR experiments and sequencing results. In general, with the ever-increasing amount of data that is available, our method has a strong time cost advantage, and provides a new approach for primer design and sequence analysis.

Supplementary Materials: The following are available online at http://www.mdpi.com/1660-4601/17/16/5958/s1, Table S1. napA Single Primer Result, Table S2. napA Primer Sets, Table S3. AOA Single Primer Result, Table S4. AOA Primer Sets, Table S5. AOB Single Primer Result, Table S6. AOB Primer Sets. Table S7. Calculation time comparison. Figure S1. Amplification results of primer set AOB_kmer2/AOB_kmer28 in soil samples.

Author Contributions: Conceptualization, Y.D. and Y.W.; methodology, Y.D. and Y.W.; software, K.F. and Y.W.; validation, Z.W. (Ziyan Wei), Z.W. (Zhujun Wang) and Y.W.; formal analysis, Z.W. (Zhujun Wang) and Y.W; data curation, K.F. and Y.W.; writing-original draft preparation, Y.W.; writing-review and editing, Y.D.; visualization, K.F. and Y.W.; supervision, Y.D.; project administration, Y.D.; funding acquisition, Y.D. All authors have read and agreed to the published version of the manuscript.

Funding: This research was funded by the National Nature Science Foundation of China, grant number U1906223 and 91851106, the Key Research Program of Frontier Sciences, CAS, grant number QYZDB-SSW-DQC026.

Acknowledgments: We gratefully thank James W. Voordeckers for his suggestions and grammar corrections on this paper. 
Conflicts of Interest: The authors declare no conflict of interest.

\section{References}

1. Gilbert, J.A.; Meyer, F.; Jansson, J.; Gordon, J.; Pace, N.; Tiedje, J.; Ley, R.; Fierer, N.; Field, D.; Kyrpides, N. The earth microbiome project: Meeting report of the "1 st EMP meeting on sample selection and acquisition" at Argonne National Laboratory October 6th 2010. Stand. Genom. Sci. 2010, 3, 249-253. [CrossRef] [PubMed]

2. Hugenholtz, P. Exploring prokaryotic diversity in the genomic era. Genome Biol. 2002, 3, 1-8. [CrossRef] [PubMed]

3. Pettersson, E.; Lundeberg, J.; Ahmadian, A. Generations of sequencing technologies. Genomics 2009, 93, 105-111. [CrossRef] [PubMed]

4. Scharf, S.; Horn, G.; Erlich, H. Direct cloning and sequence analysis of enzymatically amplified genomic sequences. Science 1986, 233, 1076-1078. [CrossRef]

5. Pace, N.R. Analyzing natural microbial populations by rRNA sequences. ASM News 1985, 51, 4-12.

6. Li, S.; Deng, Y.; Wang, Z.; Zhang, Z.; Kong, X.; Zhou, W.; Yi, Y.; Qu, Y. Exploring the accuracy of amplicon-based internal transcribed spacer markers for a fungal community. Mol. Ecol. Resour. 2020, 20, 170-184. [CrossRef]

7. Linhart, C.; Shamir, R. The degenerate primer design problem. Bioinformatics 2002, 18, S172-S181. [CrossRef]

8. Walters, W.A.; Caporaso, J.G.; Lauber, C.L.; Berg-Lyons, D.; Fierer, N.; Knight, R. PrimerProspector: De novo design and taxonomic analysis of barcoded polymerase chain reaction primers. Bioinformatics 2011, 27, 1159-1161. [CrossRef]

9. Hugerth, L.W.; Wefer, H.A.; Lundin, S.; Jakobsson, H.E.; Lindberg, M.; Rodin, S.; Engstrand, L.; Andersson, A.F. DegePrime, a program for degenerate primer design for broad-taxonomic-range PCR in microbial ecology studies. Appl. Environ. Microbiol. 2014, 80, 5116-5123. [CrossRef]

10. Edgar, R.C. MUSCLE: Multiple sequence alignment with high accuracy and high throughput. Nucleic Acids Res. 2004, 32, 1792-1797. [CrossRef]

11. Benoit, G.; Peterlongo, P.; Mariadassou, M.; Drezen, E.; Schbath, S.; Lavenier, D.; Lemaitre, C. Multiple comparative metagenomics using multiset k-mer counting. PeerJ Comput. Sci. 2016, 2, e94. [CrossRef]

12. Ward, T.; Larson, J.; Meulemans, J.; Hillmann, B.; Lynch, J.; Sidiropoulos, D.; Spear, J.; Caporaso, G.; Blekhman, R.; Knight, R. BugBase predicts organism level microbiome phenotypes. BioRxiv 2017, 133462.

13. Marçais, G.; Kingsford, C. A fast, lock-free approach for efficient parallel counting of occurrences of k-mers. Bioinformatics 2011, 27, 764-770. [CrossRef] [PubMed]

14. Zuker, M. Mfold web server for nucleic acid folding and hybridization prediction. Nucleic Acids Res. 2003, 31, 3406-3415. [CrossRef] [PubMed]

15. Shi, Z.; Yin, H.; Van Nostrand, J.D.; Voordeckers, J.W.; Tu, Q.; Deng, Y.; Yuan, M.; Zhou, A.; Zhang, P.; Xiao, N. Functional Gene Array-Based Ultrasensitive and Quantitative Detection of Microbial Populations in Complex Communities. MSystems 2019, 4. [CrossRef]

16. Finn, R.D.; Clements, J.; Eddy, S.R. HMMER web server: Interactive sequence similarity searching. Nucleic Acids Res. 2011, 39, W29-W37. [CrossRef]

17. Sayers, E.W.; Beck, J.; Brister, J.R.; Bolton, E.E.; Canese, K.; Comeau, D.C.; Funk, K.; Ketter, A.; Kim, S.; Kimchi, A. Database resources of the national center for biotechnology information. Nucleic Acids Res. 2020, 48, D9. [CrossRef]

18. Fish, J.A.; Chai, B.; Wan, Q.; Sun, Y.; Titus Brown, C.; Tiedje, J.M.; Cole, J.R. FunGene: The functional gene pipeline and repository. Front. Microbiol. 2013, 4, 291. [CrossRef]

19. Katoh, K.; Asimenos, G.; Toh, H. Multiple alignment of DNA sequences with MAFFT. In Bioinformatics for DNA Sequence Analysis; Springer: Boston, MA, USA, 2009; pp. 39-64.

20. Mancabelli, L.; Milani, C.; Lugli, G.A.; Fontana, F.; Turroni, F.; van Sinderen, D.; Ventura, M. The Impact of Primer Design on Amplicon-Based Metagenomic Profiling Accuracy: Detailed Insights into Bifidobacterial Community Structure. Microorganisms 2020, 8, 131. [CrossRef]

21. Wei, Z.; Feng, K.; Li, S.; Zhang, Y.; Chen, H.; Yin, H.; Xu, M.; Deng, Y. Exploring abundance, diversity and variation of a widespread antibiotic resistance gene in wastewater treatment plants. Environ. Int. 2018, 117, 186-195. [CrossRef] 
22. Sievers, F.; Wilm, A.; Dineen, D.; Gibson, T.J.; Karplus, K.; Li, W.; Lopez, R.; McWilliam, H.; Remmert, M.; Söding, J. Fast, scalable generation of high-quality protein multiple sequence alignments using Clustal Omega. Mol. Syst. Biol. 2011, 7, 539. [CrossRef] [PubMed]

23. Tu, Q.; Lin, L.; Cheng, L.; Deng, Y.; He, Z. NCycDB: A curated integrative database for fast and accurate metagenomic profiling of nitrogen cycling genes. Bioinformatics 2018, 35, 1040-1048. [CrossRef] [PubMed]

(C) 2020 by the authors. Licensee MDPI, Basel, Switzerland. This article is an open access article distributed under the terms and conditions of the Creative Commons Attribution (CC BY) license (http://creativecommons.org/licenses/by/4.0/). 